\title{
Synthesis, spectral and B3LYP analysis of 1-R-2-oxo-pyrido[2,1b][3,4]dihydro-pyrimidinium halides
}

\author{
Iwona Kowalczyk and Mirosław Szafran* \\ Adam Mickiewicz University, Poznan, Grunwaldzka 6, Poland \\ E-mail:szafran@amu.edu.pl
}

Dedicated to Professor Jan Epsztajn on his $75^{\text {th }}$ birthday

\begin{abstract}
2-NHR-Pyridines $\left(\mathrm{R}=\mathrm{H}, \mathrm{CH}_{3}\right)$ on the reaction with 3-halogenopropionic acids have been found to give 1-R-2-oxo-pyrido[2,1b][3,4]dihydro-pyrimidinium halides $(\mathbf{1}(\mathrm{R}=\mathrm{Me}), 2(\mathrm{R}=\mathrm{H})$. Their structures have been confirmed by the ${ }^{1} \mathrm{H},{ }^{13} \mathrm{C}$ NMR spectra in DMSO- $\mathrm{d}_{6}$ solution using 2D NMR techniques (HETCOR, COSY, HMBC), FTIR spectra and B3LYP/6-31G(d,p) calculations. Only 2 on the reaction with propylene oxide is converted into betaine, 3-(2-aminopyridinium)-propionate (4). This suggest that in solution $1-H$-2-oxo-pyrido[2,1b][3,4]dihydropyrimidinium chloride is in an equilibrium with $N$-carboxyethyl-2-amino-pyridinium chloride ( 2 $\leftrightarrows 3)$
\end{abstract}

Keywords: 1 - $R$-2-Oxo-pyrido[2,1 $b][3,4]$ dihydro-pyrimidinium halides, 3-(2-amino-pyridinium)propionate, NMR and FTIR spectra, DFT calculations, conformation of pyrimidinium ring

\section{Introduction}

Quaternization of six-membered heteroaromatic compounds has been carried out with a wide range of alkylation agents. ${ }^{1-9}$ Several $N$-phenacylpyridinium halides have been the focus of a great deal of attention. ${ }^{10-15}$ Acidic hydrogens of the $\mathrm{N}^{+}-\mathrm{CH}_{2}$ group are easily exchangeable for deuterium in $\mathrm{D}_{2} \mathrm{O}$ and can be converted into highly reactive ylides. ${ }^{16}$ Pyridines with $\alpha$ substituents usually are slightly less reactive, which is probably caused by steric factors. ${ }^{10}$ Several 2-substituted pyridines $\left(\mathrm{CN}, \mathrm{CH}=\mathrm{NOH}, \mathrm{CH}_{2} \mathrm{OH}, \mathrm{COCH}_{2} \mathrm{R}, \mathrm{CONH}_{2}, \mathrm{COOEt}\right.$, etc.) during quaternization immediately cyclized to pyrido[2,1c]pyrazinium or pyrido[2,1c]oxazinium halides. $^{17-21}$

Recently, we have described the synthesis and the crystal structure of 3-hydroxy-3-phenylpyrido[2,1-c][1,4]dihydrooxazinium bromide, which in DMSO and $\mathrm{D}_{2} \mathrm{O}$ solutions exist in an equilibrium with N-phenacyl-2-hydroxymethyl-pyridinium bromide (Scheme 1). ${ }^{22}$ 

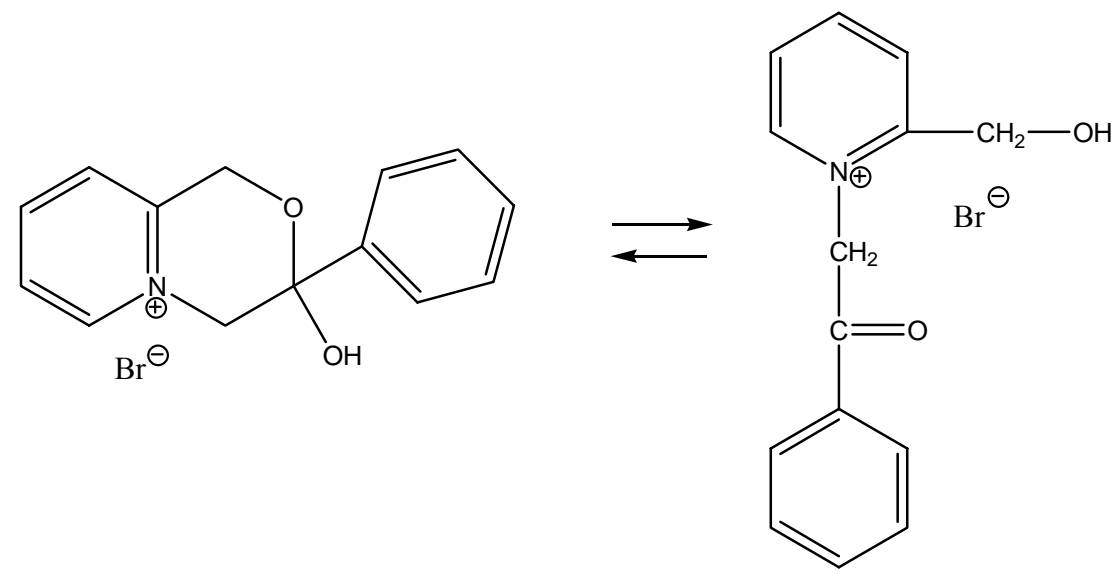

Scheme 1. An equilibrium between 3-hydroxy-3-phenyl-pyrido[2,1-c][1,4]dihydrooxazinium and $N$-phenacyl-2-hydroxymethyl-pyridinium bromides.

In this work we describe the reactions of $2-\mathrm{NH}_{2-}$ and 2-NHMe-pyridines with 3halogenopropionic acids using the procedure developed by Kröhnke et al. ${ }^{17}$ and analyze structures of the compounds obtained.

\section{Results and Discussion}

Quaternization of 2-NHMe-pyridine with 3-bromopropionic acids gave 1-Me-2-oxopyrido[2,1b][3,4]dihydro-pyrimidinium bromide (1), which does not react with propylene oxide (Scheme 2). Both ${ }^{1} \mathrm{H}$ and ${ }^{13} \mathrm{C}$ NMR spectra of $\mathbf{1}$ are typical for pure compound and suggests that in solution it exists in a cyclic structure. The $v \mathrm{C}=\mathrm{O}$ band at $1714 \mathrm{~cm}^{-1}$ and the lack of the $v \mathrm{NH}$ band above $2000 \mathrm{~cm}^{-1}$ in the IR spectrum confirm the cyclic structure of $\mathbf{1}$. Additionally the ${ }^{1} \mathrm{H}^{13} \mathrm{C}$ HMBC spectrum (Figure 1) shows a three bond correlation between the $\mathrm{N}-\mathrm{CH}_{3}$ protons and $\mathrm{C}=\mathrm{O}$ carbon in compound $\mathbf{1}$.

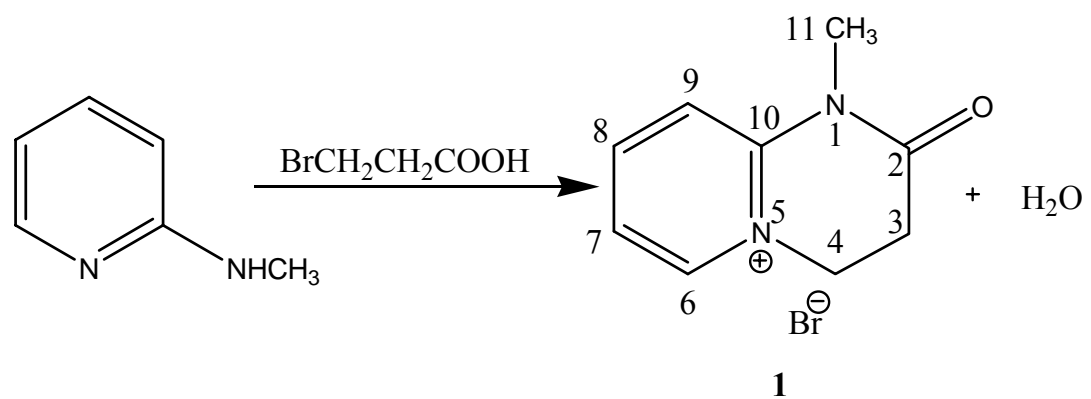

Scheme 2. Synthesis of 1-Me-2-oxo-pyrido[2,1b][3,4]-dihydro-pyrimidinium bromide (1). 


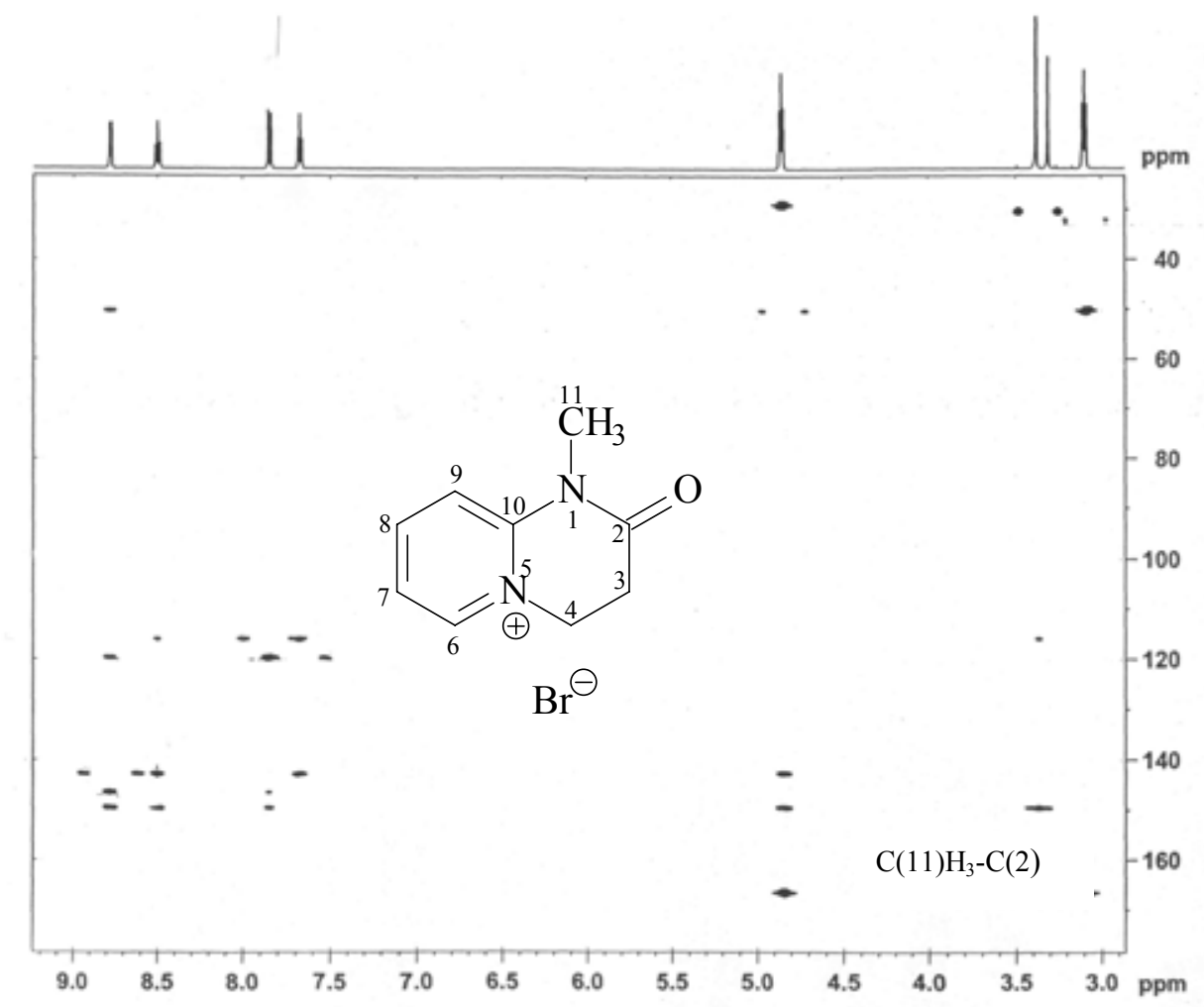

Figure 1. ${ }^{1} \mathrm{H}^{13} \mathrm{C}$ HMBC spectrum of 1-Me-2-oxo-pyrido[2,1b][3,4]dihydro-pyrimidinium bromide (1) in DMSO-d 6 .

2-Aminopyridine with 3-chloropropionic acid gave 1- $H$-2-oxo-pyrido[2,1b][3,4]dihydropyrimidinium chloride (2). The FTIR spectrum in solid state shows the $v \mathrm{NH}$ band at $2657 \mathrm{~cm}^{-1}$ and two $v \mathrm{C}=\mathrm{O}$ bands at 1706 and $1723 \mathrm{~cm}^{-1}$. Two $v \mathrm{C}=\mathrm{O}$ bands in the spectrum suggest the presence of two conformers in the crystal. The ${ }^{1} \mathrm{H}$ and ${ }^{13} \mathrm{C}$ NMR spectra in $\mathrm{DMSOd}_{6}$ (Figure 2) are typical of mixture of $\mathbf{2}$ (main component) and 3-(2-amino-pyridinium)-propionate hydrochloride, $\mathbf{3}$ (small amount). 


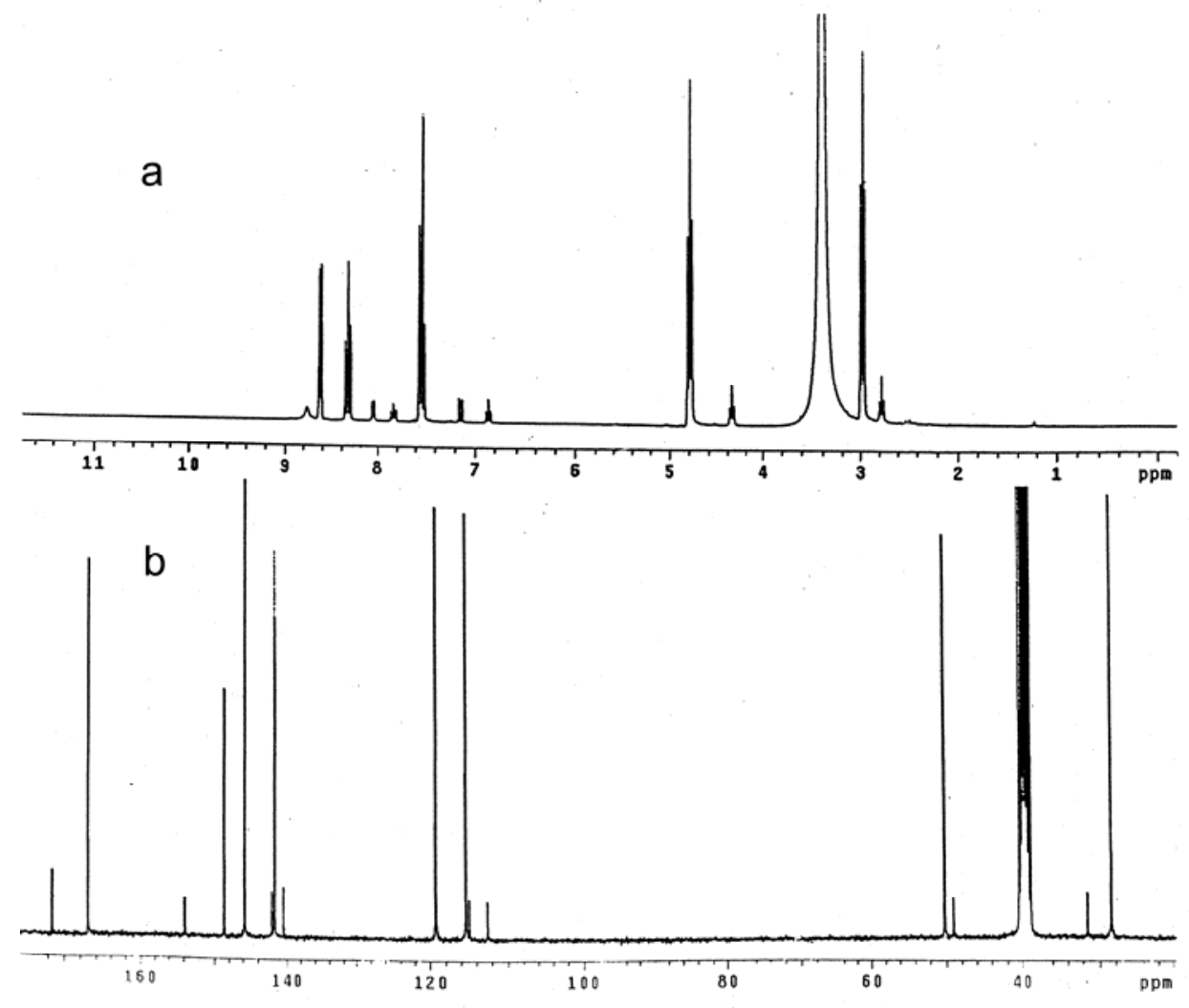

Figure 2. The NMR spectra of 1-H-2-oxo-pyrido[2,1b][3,4]dihydro-pyrimidinium chloride (2) in DMSO-d $_{6}$, a) ${ }^{1} \mathrm{H}$ NMR spectrum ; b) ${ }^{13} \mathrm{C}$ NMR spectrum.

Conversion of $\mathbf{3}$ with propylene oxide into 3-(2-amino-pyridinium)-propionate (4) confirmed an equilibrium $\mathbf{2} \leftrightarrows \mathbf{3}$ (Scheme 3). Propylene oxide was used to abstract hydrochloride from betaine hydrochlorides. ${ }^{23,24}$ Mechanism of this reaction is probably similar to the reaction of propylene oxide with aqueous hydrochloric acid. ${ }^{25}$ Carboxylic acids, and probably amides, are less reactive with oxiranes and require catalyst. ${ }^{26,27}$ Thus reaction of 2 with propylene oxide is less probable.

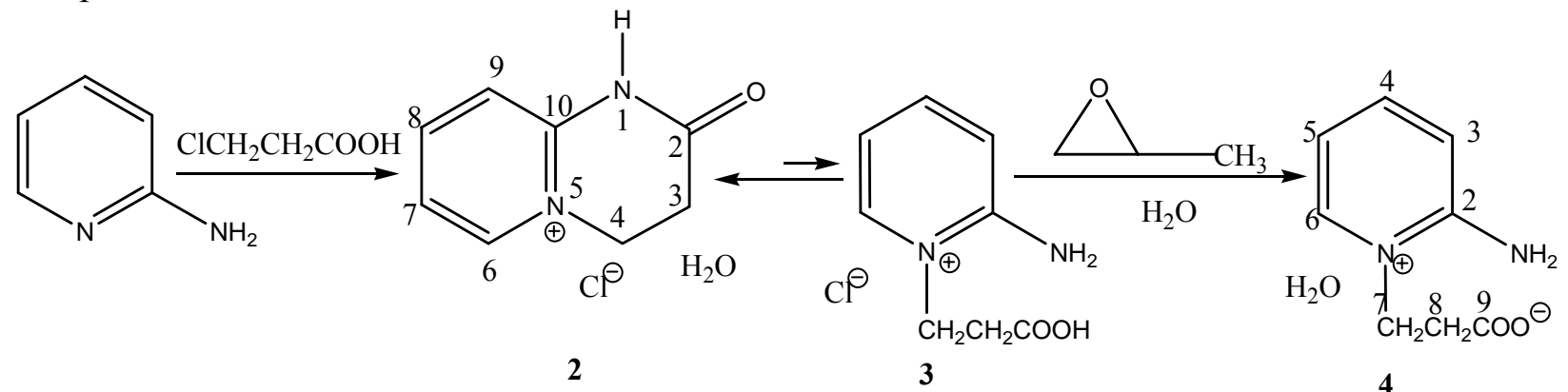

Scheme 3. Synthesis of 1-H-2-oxo-pyrido[2,1b][3,4]dihydro-pyrimidinium chloride (2) and 3(2-amino-pyridinium)-propionate (4). 
Two conformers of 1-Me-2-oxo-pyrido[2,1b][3,4]dihydro-pyrimidinium bromide, denoted as $\mathbf{1 a}$ and $\mathbf{1 b}$ and two conformers of 1-H-2-oxo-pyrido[2,1b][3,4]dihydro-pyrimidinium chloride denoted as $\mathbf{2 a}$ and $\mathbf{2 b}$ were optimized by the B3LYP/631G(d,p) level of theory and selected data are listed in Table 1. Their structures are shown in Figure 3. Conformers a and b have, respectively, very similar energy, slightly different dipole moments, but the opposite sign of the $\mathrm{N}(5)-\mathrm{C}(4)-\mathrm{C}(3)-\mathrm{C}(2)$ dihedral angle. The energy barrier between $\mathbf{a}$ and $\mathbf{b}$ cations, calculated by the PM3 method using the B3LYP/6-31G(d,p) geometries, is 4.9 and $1.7 \mathrm{kcal} / \mathrm{mol}$ for $\mathbf{1}$ and $\mathbf{2}$, respectively (Figure 4).

Table 1. Selected parameters of conformers 1a-b and $\mathbf{2 a - b}$ estimated by B3LYP/6-31G(d,p) calculations

\begin{tabular}{|l|l|l|l|l|}
\hline \multicolumn{1}{|c|}{ Parameter } & \multicolumn{4}{c|}{ Conformer } \\
\hline & \multicolumn{1}{|c|}{$\mathbf{1 a}$} & \multicolumn{1}{c|}{$\mathbf{1 b}$} & \multicolumn{1}{c|}{$\mathbf{2 a}$} & \multicolumn{1}{c|}{$\mathbf{b}$} \\
\hline Energy (a.u) & -3106.042687 & -3106.042688 & -952.221649 & -952.221649 \\
\hline Dip. Moment (D) & 7.8254 & 7.8206 & 15.2888 & 15.2873 \\
\hline $\mathrm{C}(2)=\mathrm{O}(1)(\tilde{\mathrm{A}})$ & 1.215 & 1.215 & 1.210 & 1.210 \\
\hline $\mathrm{N}(1) \cdots \mathrm{Br} / \mathrm{Cal}$ & 4.843 & 4.843 & 2.893 & 2.893 \\
\hline $\mathrm{N}-\mathrm{H}$ & & & 1.135 & 1.135 \\
\hline $\mathrm{H} \cdots \mathrm{Cal}$ & & & 1.777 & 1.777 \\
\hline $\mathrm{N}-\mathrm{H} \cdot \mathrm{Cl}(\mathrm{deg})$. & & & 166.6 & 166.6 \\
\hline $\mathrm{N}(5) \cdots \mathrm{Br} / \mathrm{Cl}(\tilde{\mathrm{A}})$ & 3.165 & 3.165 & 4.887 & 4.887 \\
\hline $\mathrm{N}(5)-\mathrm{C}(4)-\mathrm{C}(3)-\mathrm{C}(2)(\mathrm{deg})$ & 56.12 & -56.12 & 53.04 & -53.04 \\
\hline $\mathrm{C}(10)-\mathrm{N}(5)-\mathrm{C}(4)-\mathrm{C}(3)$ & -36.91 & 36.94 & -38.46 & 38.44 \\
\hline $\mathrm{C}(6)-\mathrm{N}(5)-\mathrm{C}(4)-\mathrm{C}(3)$ & 129.07 & -129.00 & 146.10 & -146.10 \\
\hline $\mathrm{N}(1)-\mathrm{C}(2)-\mathrm{C}(3)-\mathrm{C}(4)$ & -35.67 & 35.53 & -36.53 & 36.52 \\
\hline
\end{tabular}




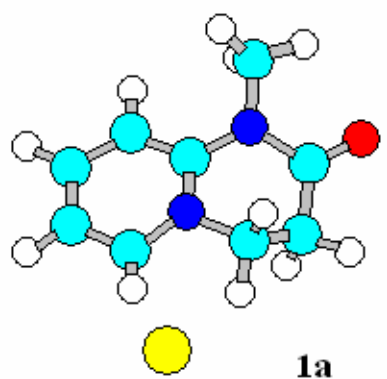

$1 \mathbf{a}$

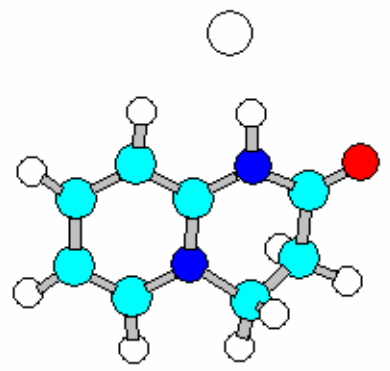

$2 \mathbf{a}$

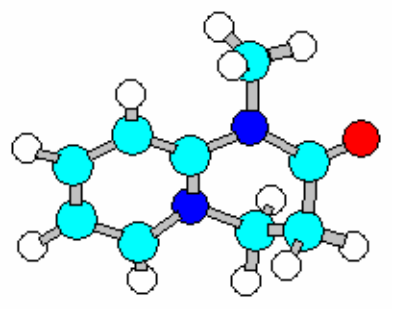

1b

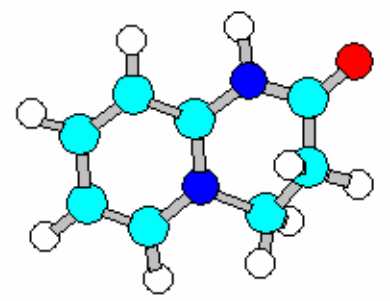

2b

Figure 3. Structures of conformers 1-methyl-2-oxo-pyrido[2,1b][3,4]dihydro-pyrimidinium bromide (1a-b) and 1-H-2-oxo-pyrido[2,1b][3,4]dihydro-pyrimidinium chloride (2a-b) calculated by the B3LYP/6-31G(d,p) method.
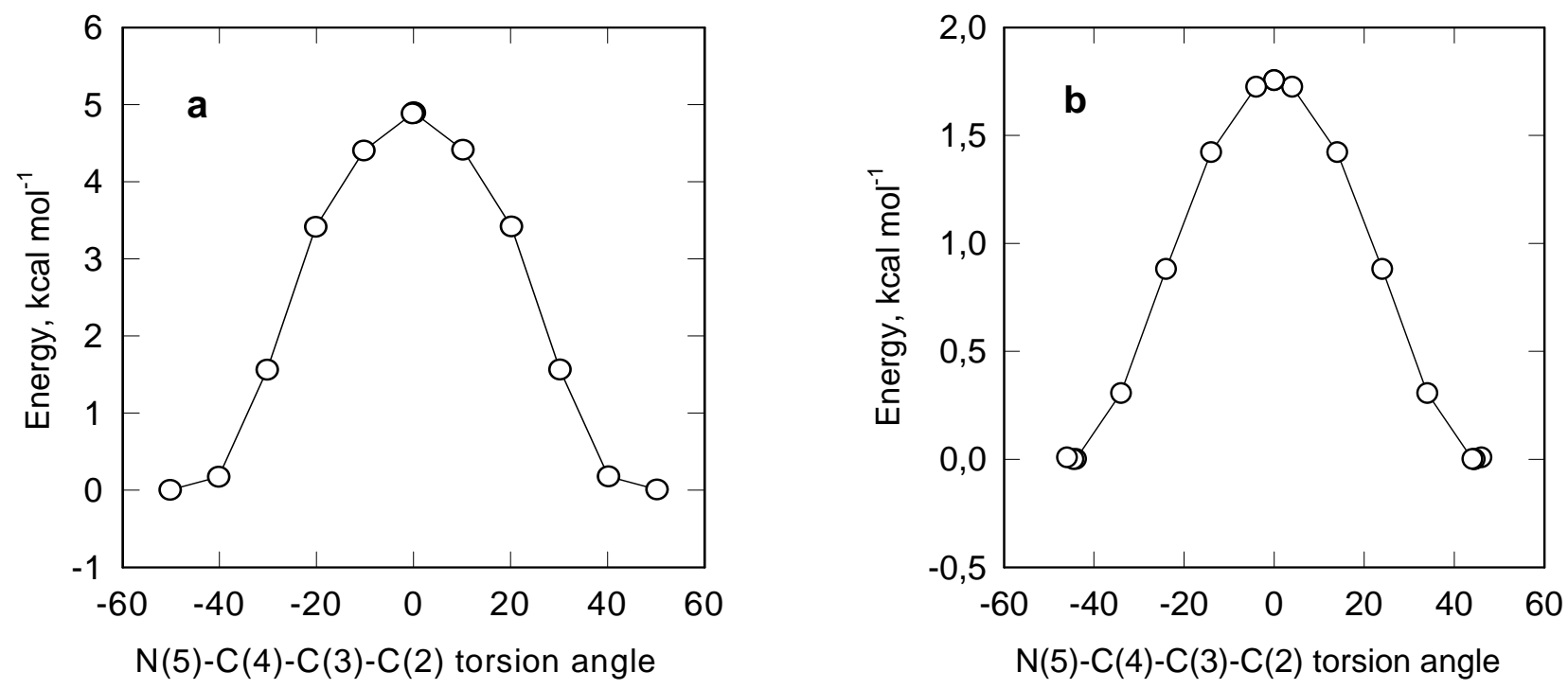

Figure 4. Potential energy as a function of the $\mathrm{N}(5)-\mathrm{C}(4)-\mathrm{C}(3)-\mathrm{C}(2)$ torsion angle: a) 1-methyl-2oxo-pyrido[2,1b][3,4]dihydro-pyrimidinium bromide, $\mathbf{1}$ and b) 1-H-2-oxopyrido[2,1b][3,4]dihydro-pyrimidinium chloride, 2 . 


\section{Experimental Section}

General Procedures. All NMR spectra were measured with a Varian Gemini 300 VT spectrometer, operating at 300.07 and $75.4614 \mathrm{~Hz}$ for ${ }^{1} \mathrm{H}$ and ${ }^{13} \mathrm{C}$, respectively. Typical conditions for the proton spectra were: pulse width $32^{\circ}$, acquisition time $5 \mathrm{~s}$, FT size $32 \mathrm{~K}$ and digital resolution $0.3 \mathrm{~Hz}$ per point, and for the carbon spectra pulse width $60^{\circ}$, FT size $60 \mathrm{~K}$ and digital resolution $0.6 \mathrm{~Hz}$ per point, the number of scans varied from 1200 to 10000 per spectrum. The ${ }^{13} \mathrm{C}$ and ${ }^{1} \mathrm{H}$ chemical shifts were measured in DMSO- $\mathrm{d}_{6}$ and are reported in $\delta(\mathrm{ppm})$ relative to an internal standard of tetramethylsilane (TMS). All proton and carbon-13 resonances were assigned by ${ }^{1} \mathrm{H}(\mathrm{COSY}),{ }^{13} \mathrm{C}$ (HETCOR, HMBC). The FTIR spectra were recorded in Nujol and Florolube mulls at $2 \mathrm{~cm}^{-1}$ resolution on a Bruker IFS $113 \mathrm{v}$ spectrometer, which was evacuated to avoid water and $\mathrm{CO}_{2}$ absorption. Each spectrum consists of 250 scans at $31^{\circ} \mathrm{C}$. The calculations were performed using the GAUSSIAN 03 package $^{28}$ at the B3LYP level of theory ${ }^{29-}$ ${ }^{31}$ and the $6-31 \mathrm{G}(\mathrm{d}, \mathrm{p})$ basis $\operatorname{set}^{32}$.

1-Methyl-2-oxo-pyrido[2,1b][3,4]dihydro-pyrimidinium bromide (1). 2-Methylaminopyridine $(2.19 \mathrm{~g}, 0.02 \mathrm{M})$ and 3-bromopropionic acid $(3.06 \mathrm{~g}, 0.02 \mathrm{M})$ in $20 \mathrm{ml}$ acetonitrile were stirred at room temperature up to solification and then left to stand for 2 days. The solvent was evaporated under reduced pressure and the residue was dried over $\mathrm{P}_{4} \mathrm{O}_{10}$ and then recrystallized from anhydrous methanol, yield 84\%, m.p. $281-282{ }^{\circ} \mathrm{C}$. Analysis: found (calc) $\% \mathrm{C} 44.45$ (44.47); \%H 4.70 (4.56); \%N 11.54 (11.52). ${ }^{1} \mathrm{H}$ NMR (DMSO-d 6 ): $\delta 3.38\left(3 \mathrm{H}, \mathrm{s}, \mathrm{C}(11) \mathrm{H}_{3}\right), 3.09$ $\left(2 \mathrm{H}, \mathrm{t}, \mathrm{C}(3) \mathrm{H}_{2}\right), 4.85\left(2 \mathrm{H}, \mathrm{t}, \mathrm{C}(4) \mathrm{H}_{2}\right), 7.85(1 \mathrm{H}, \mathrm{d}, \mathrm{C}(9) \mathrm{H}), 8.50(1 \mathrm{H}, \mathrm{t}, \mathrm{C}(8) \mathrm{H}), 7.68(1 \mathrm{H}, \mathrm{t}$, $\mathrm{C}(7) \mathrm{H}), 8.78(1 \mathrm{H}, \mathrm{d}, \mathrm{C}(6) \mathrm{H}) ;{ }^{13} \mathrm{C}$ NMR (DMSO-d 6$): \delta 30.4(\mathrm{C}(11)), 28.9(\mathrm{C}(3)), 50.1(\mathrm{C}(4))$, $149.2(\mathrm{C}(10)), 115.7(\mathrm{C}(9)), 142.5(\mathrm{C}(8)), 119.5(\mathrm{C}(7)), 146.1(\mathrm{C}(6)), 166.4(\mathrm{C}(2))$; FTIR: $v(\mathrm{C}=\mathrm{O})$ at $1714 \mathrm{~cm}^{-1} ; \mathrm{ES}^{+} \mathrm{MS} \mathrm{m} / \mathrm{z} 163\left(\mathrm{C}_{9} \mathrm{H}_{11} \mathrm{~N}_{2} \mathrm{O}\right)$.

2-Oxo-pyrido[2,1b][3,4]dihydro-pyrimidinium chloride (2). 2-Amino-pyridine (1.88 g, 0.02 $\mathrm{M})$ and 3-chloropropionic acid $(1.89 \mathrm{~g}, 0.02 \mathrm{M})$ in $20 \mathrm{ml}$ acetonitrile were stirred at room temperature up to solification and then left to stand for 10 days. The solvent was evaporated under reduced pressure and the residue was dried over $\mathrm{P}_{4} \mathrm{O}_{10}$ and then recrystallized from anhydrous ethanol, yield 73\%, m.p. $278-279{ }^{\circ} \mathrm{C} ;{ }^{1} \mathrm{H}$ NMR (DMSO-d $\left.\mathrm{d}_{6}\right): \delta 2.98\left(2 \mathrm{H}, \mathrm{t}, \mathrm{C}(3) \mathrm{H}_{2}\right)$, $4.81\left(2 \mathrm{H}, \mathrm{t}, \mathrm{C}(4) \mathrm{H}_{2}\right), 7.64$ ( 1 H, d, C $\left.(9) \mathrm{H}\right), 8.34(1 \mathrm{H}, \mathrm{t}, \mathrm{C}(8) \mathrm{H}), 7.54(1 \mathrm{H}, \mathrm{t}, \mathrm{C}(7) \mathrm{H}), 8.66(1 \mathrm{H}, \mathrm{d}$, $\mathrm{C}(6) \mathrm{H}) ;{ }^{13} \mathrm{C}$ NMR (DMSO-d 6 ): $\delta 28.5(\mathrm{C}(3), 50.1(\mathrm{C}(4)), 148.4(\mathrm{C}(10)), 115.2(\mathrm{C}(9)), 141.5$ $(\mathrm{C}(8)), 119.0(\mathrm{C}(7)), 145.5(\mathrm{C}(6)), 166.7(\mathrm{C}(2))$; FTIR: $v(\mathrm{C}=\mathrm{O})$ at 1723 and $1706 \mathrm{~cm}^{-1}, v \mathrm{NH}$ at $2657 \mathrm{~cm}^{-1}$ as a broad band; $\mathrm{ES}^{+} \mathrm{MS} \mathrm{m} / \mathrm{z} 149\left(\mathrm{C}_{8} \mathrm{H}_{9} \mathrm{~N}_{2} \mathrm{O}\right)$.

3-(2-Amino-pyridinium)propionate monohydrate (4). 2-oxo-pyrido[2,1b][3,4]dihydropyrimidinium chloride $(3.68 \mathrm{~g}, 0.02 \mathrm{M})$ was dissolved in water and propylene oxide $(1.45 \mathrm{~g}$, $0.025 \mathrm{M}$ ) was added slowly and left for 4 days. To remove chloropropanols, the reaction mixture was extracted six times with ether and the residue was evaporated under reduced pressure to dryness. The raw product was recrystallized from methanol, m.p. $124-126{ }^{\circ} \mathrm{C}$, yield $63 \%$. ${ }^{1} \mathrm{H}$ NMR (DMSO-d $\left.{ }_{6}\right): \delta 2.54\left(2 \mathrm{H}, \mathrm{t}, \mathrm{C}(8) \mathrm{H}_{2}\right), 4.18\left(2 \mathrm{H}, \mathrm{t}, \mathrm{C}(7) \mathrm{H}_{2}\right), 6.83(1 \mathrm{H}, \mathrm{d}, \mathrm{C}(3) \mathrm{H}), 7.60(1 \mathrm{H}$, 
t, $\mathrm{C}(4) \mathrm{H}), 6.69(1 \mathrm{H}, \mathrm{t}, \mathrm{C}(5) \mathrm{H}), 7.65(1 \mathrm{H}, \mathrm{d}, \mathrm{C}(6) \mathrm{H}) .{ }^{13} \mathrm{C}$ NMR (DMSO-d $\left.\mathrm{d}_{6}\right): \delta 35.8(\mathrm{C}(8)), 51.9$ (C(7)), $154.7(\mathrm{C}(2)), 114.5(\mathrm{C}(3)), 140.4(\mathrm{C}(4)), 115.9(\mathrm{C}(5)), 143.4(\mathrm{C}(6)), 178.5$ (C(9)); FTIR: $v(\mathrm{COO})$ at $1696 \mathrm{~cm}^{-1}, \mathrm{v}\left(\mathrm{NH}_{2}+\mathrm{H}_{2} \mathrm{O}\right)$ in $3500-2600 \mathrm{~cm}^{-1}$ region.

\section{Acknowledgements}

DFT calculations were performed at the Poznań Supercomputing and Networking Centre.

\section{References}

1. Jones, R.A.; Quaternary Ammonium Salts. Their Use in Phase-Transfer Catalysed Reactions, Academic Press: San Diego, 2001.

2. Śliwa, W. Heterocycles 1989, 29, 557.

3. Zoltewicz, J.; Deady, L. W. Adv. Heterocycl. Chem. 1978, $2,71$.

4. Forster, W.; Laird, R. M. J. Chem. Soc. Perkin Trans. 2 1991, 1033.

5. Van der Plas, H.C.; Heterocycles 1978, 9, 33.

6. Ośmiałowski, B.; Janota, H.; Gawinecki, R.; Polish J. Chem. 2003, 7, 169, and ref. cited therein.

7. Carey, A. R. E.; More - O'Ferall, R. A.; Murray, B. A.; J. Chem. Soc., Perkin Trans 2 1993, 2297.

8. Litvinov, V. P.; Shestopalov, A. M.; Zh. Org. Khim. (in Russian) 1997, 33, 975, and references cited therein.

9. Pernak, J.; Kalewska, J.; Ksycińska, H.; Cybulski, J. Eur. J. Med. Chem. 2001, 36, 899.

10. Barbieri, G.; Benassi, R.; Grandi, R.; Pagnoni, U. M.; Taddei, F. Org. Magn. Reson. 1979, $12,159$.

11. Henrick, C. A.; Ritchie, E.; Taylor, W. C. Aust. J. Chem. 1967, 20, 2441.

12. Surpateanu, G. Heterocycles 1984, 22, 2079

13. Clark, J. S.; (editor) Nitrogen, Oxygen and Sulfur Ylide Chemistry, Oxford University Press, 2002.

14. Phillips, W. G.;Ratts, K. W. J. Org. Chem. 1970, 35, 3144.

15. Rozas, I.; Alkorta, I.; Elguero, J. J. Am. Chem. Soc. 2000, 122, 11154.

16. Szafran, M.; Szwajca, A. Polish J. Chem. 2004, 78, 2131.

17. Kröhnke, F.; Schnegelberger, H.; Weis, W. Chem. Ber. 1964, 97, 3566.

18. Adamson, J.; Glover, E. E. J. Chem. Soc. C 1971, 861.

19. Bátori, S.; Messmer, A.; J. Heterocyclic Chem. 1990, 27, 1673.

20. Hermecz, I. Adv. Heterocyclic Chem. 2003, 85, 173.

21. Szafran, M.; Nowak-Wydra, B.; Jaskólski, M.; Bartoszak-Adamska, E.; Szwajca, A.; DegaSzafran, Z. J. Mol. Struct. 2005, 743, 7. 
22. Szafran, M.; Nowak-Wydra, B.; Katrusiak, A.; Dega-Szafran, Z. J. Mol. Struct. in press.

23. Szafran, M.; Kowalczyk, I.; Katrusiak, A. J. Mol. Struct. 2006, 786, 25.

24. Szafran, M.; Katrusiak, A.; Dega-Szafran, Z. J. Mol. Struct. in press.

25. Phillips, R. E.; Soulen, R. L. J. Chem. Edu. 1995, 72, 624.

26. Bukowska, K.; Bukowski, W.; Galina, H. Wiadomości Chemiczne 1997, 51, 217 and references therein.

27. Weissermel, K.; Arpe, H. J. Industrial Organic Chemistry, VCH: Weinheim, 1993.

28. Frisch, M. J.; Trucks, G. W.; Schlegel, H. B.; Scuseria, G. E.; Robb, M. A.; Cheeseman, J. R.; Montgomery, J. A.; Vreven, Jr. T.; Kudin, K. N.; Burant, J. C.; Millam, J. M.; Iyengar, S. S.; Tomasi, J.; Barone, V.; Mennucci, B.; Cossi, M.; Scalmani, G.; Rega, N.; Petersson, G. A.; Nakatsuji, H.; Hada, M.; Ehara, M.; Toyota, K.; Fukuda, R.; Hasegawa, J.; Ishida, M.; Nakajima, T.; Honda, Y.; Kitao, O.; Nakai, H.; Klene, M. X. Li.; Knox, J. E.; Hratchian, H. P.; Cross, J. B.; Adamo, C.; Jaramillo, J.; Gomperts, R.; Stratmann, R. E.; Yazyev, O.; Austin, A. J.; Cammi, R.; Pomelli, C.; Ochterski, J. W.; Ayala, P. Y.; Morokuma, K.; Voth, G. A.; Salvador, P.; Dannenberg, J. J.; Zakrzewski, V. G.; Dapprich, S.; Daniels, A. D.; Strain, M. C.; Farkas, O.; Malick, D. K.; Rabuck, A. D.; Raghavachari, K.; Foresman, J. B.; Ortiz, J. V.; Cui, Q.; Baboul, A. G.; Clifford, S.; Cioslowski, J.; Stefanov, B. B.; Liu G., Liashenko, A.; Piskorz, P.; Komaromi, I.; Martin, R. L.; Fox, D. J.; Keith, T.; Al-Laham, M. A.; Peng, C.Y.; Nanayakkara, A.; Challacombe, M.; Gill, P. M. W.; Johnson, B.; Chen, W.; Wong, M. W.; Gonzalez, C.; Pople, J. A. Gaussian 03, Revision C.01, Gaussian, Inc., Wallingford CT, 2004.

29. Becke, A. D. J. Chem. Phys. 1993, 98, 5648.

30. Becke, A. D. J. Chem. Phys. 1997, 107, 8554.

31. Lee, C.; Yang, W.; Parr, G. R. Phys. Rev. 1988, B 37, 785.

32. Hehre, W. J.; Random, L.; Schleyer, P.V.R.; Pople, J.A.; Ab Initio Molecular Orbital Theory, Wiley: New York, 1986. 